\title{
Adaptabilidade e estabilidade de cultivares e híbridos de milho no Nordeste brasileiro no ano agrícola de $1998^{(1)}$
}

\author{
Hélio Wilson Lemos de Carvalho( ${ }^{(2)}$, Maria de Lourdes da Silva Leal(2), Milton José Cardoso(3), \\ Manoel Xavier dos Santos ${ }^{(4)}$, Benedito Carlos Lemos de Carvalho( ${ }^{(5)}$, José Nildo Tabosa ${ }^{(6)}$, Marcelo Abdon Lira ${ }^{(7)}$ \\ e Marcondes Maurício Albuquerque ${ }^{(2)}$
}

Resumo - Durante o ano agrícola de 1998, na Região Nordeste do Brasil, foram realizados experimentos em rede, em blocos casualizados com três repetições, envolvendo a avaliação de 25 cultivares (cultivares e populações) e de 30 híbridos de milho em dezenove e dezesseis ambientes, respectivamente, objetivando conhecer adaptabilidade e estabilidade dessas cultivares para fins de recomendação. Os híbridos apresentaram maiores rendimentos, em média, 21,4\% mais que as cultivares. Nos ensaios envolvendo cultivares e populações, o híbrido Cargill 909, utilizado como testemunha, teve um rendimento superior, tanto nos ambientes desfavoráveis quanto nos favoráveis. As cultivares AL 30, BR 5028, BR 106, BR 5011, BR 5033, Sintético Dentado, Sintético Duro e a população CMS 50 expressaram boa adaptação e estabilidade de produção no Nordeste brasileiro. Nos ensaios envolvendo híbridos, Dina 1000, Cargill 333 B, Dina 270, Braskalb XL 360, Master, AG 1051, AG 4051 e Pioneer 3041 apresentaram maior adaptação com estabilidade de produção nos ambientes considerados. Para recomendação específica a ambientes favoráveis destacaram-se os híbridos AG 4051 e Pioneer X 1296 B. Apesar de não se encontrarem híbridos com adaptação específica a ambientes desfavoráveis, a recomendação de materiais com maiores rendimentos nesses ambientes é de interesse para o agricultor, a exemplo do Dina 1000, Cargill 333 B e Braskalb XL 360.

Termos para indexação: Zea mays, cultivares, interação genótipo-ambiente, adaptação.

\section{Adaptability and yield stability of corn cultivars in the Brazilian Northeast in the agricultural year of 1998}

\begin{abstract}
For the agricultural year of 1998, data from a network of experiments using a randomized block design with three replications for 25 corn cultivars and 30 hybrids cultivated in nineteen and sixteen different environments, respectively, were analysed, aiming to obtain knowledge concerning to adaptability, and yield stability of cultivars in each environment. The hybrids showed an average performance $21.4 \%$ higher than the cultivars. In the experiments involving cultivars and populations, the Cargill hybrid 909 utilized as control, had a higher yield either under unfavorable or favorable environments. The yield of AL 30, BR 5028, BR 106, BR 5011, BR 5033, "Sintético Dentado", "Sintético Duro" cultivars and the CMS 50 population expressed good adaptation and yield stability under Northeastern Brazil conditions. In the experiments with hybrids, Dina 1000, Cargill 333 B, Dina 270, Braskalb XL 360, Master, AG 1051, AG 4051 and Pioneer 3041 expressed adaptation and yield stability higher than the other hybrids tested under the different environmental conditions. At favorable environmental conditions, the hybrids AG 4051 and Pioneer X 1296 B were the best ones. Even though no hybrids were specifically indicated for unfavorable environment, the recommendation of materials showing higher yields at unfavorable environments are interesting for the corn grower. This is the case of the Dina 1000, Cargill 333 B and Braskalb XL 360.
\end{abstract}

Index terms: Zea mays, cultivars, genotype environment interaction, adaptation

\footnotetext{
(1) Aceito para publicação em 10 de julho de 2000.

(2) Embrapa-Centro de Pesquisa Agropecuária dos Tabuleiros Costeiros, Av. Beira-Mar, 3250, Caixa Postal 144, CEP 49001-970 Aracaju, SE. E-mail: helio@cpatc.embrapa.br, mma@uep.embrapa.al.gov.br

${ }^{(3)}$ Embrapa-Centro de Pesquisa Agropecuária do Meio-Norte, Caixa Postal 001 , CEP 64006-220 Teresina, PI E-mail: milton@cpamn.embrapa.br
}

(4)Embrapa-Centro Nacional de Pesquisa de Milho e Sorgo, Caixa Postal 152, CEP 35701-970 Sete Lagoas, MG. E-mail: xavier@enpms.embrapa.br

(5)Embrapa/EBDA, Av. Dorival Caymmi, 15649, CEP 41615-150 Salvador, BA. E-mail: ebdadexp@ebda.ba.gov.br (6)IPA, CEP 50761-000 Recife, PE. E-mail: tabosa@ipa.br (7)Embrapa/Emparn, Rua Major Laurentino de Moraes, 1220, CEP 59020-390 Natal, RN. 


\section{Introdução}

Considerando a extensão territorial do Nordeste brasileiro e a diversidade de ambientes existentes e de sistemas de produção praticados nos seus diferentes ecossistemas, aliadas à importância sócio-econômica de milho para a região, houve necessidade de adotar um programa de avaliação de cultivares (cultivares, populações e híbridos) de milho, visando dotar a agricultura regional de materiais de melhor adaptação. A utilização de cultivares adaptadas e portadoras de características agronômicas desejáveis, tais como, menor porte de planta e de espiga, precocidade e bom empalhamento, devem ser aconselhadas para pequenos e médios produtores, os quais em geral têm limitação de capital, que os impede de investir em tecnologias modernas de produção, além de possibilitar a reutilização de sementes em plantios posteriores. Diversos trabalhos de competição realizados no Nordeste brasileiro têm mostrado boa adaptação das cultivares melhoradas associada a boa previsibilidade de comportamento nos diferentes ambientes considerados (Cardoso et al., 1997; Monteiro et al., 1998; Carvalho et al., 1999c, 1999d).

O interesse por híbridos de milho vem aumentando gradativamente em algumas áreas da Região Nordeste, nas quais há aptidão edafoclimática para a espécie, a exemplos das áreas de cerrados nos Estados da Bahia e Maranhão, onde tem sido expressivo o uso de tecnologias modernas de produção. Outras regiões produtoras de milho do Nordeste brasileiro vêm também demandando esse tipo de material genético em razão do bom desempenho que os mesmos têm demonstrado. De fato, tem-se constatado a melhor adaptação dos híbridos em relação às cultivares melhoradas difundidas na região, em diversos trabalhos realizados no Nordeste brasileiro, nos quais os híbridos têm mostrado produtividade de $20 \%$ a $25 \%$ superior em relação às cultivares (Carvalho et al., 1999a, 1999b,1999c).

Outro fato importante a se considerar nessa vasta região é a presença da interação cultivares x ambientes, a qual assume papel fundamental no processo de recomendação de cultivares. Para que se proceda uma recomendação mais eficiente, torna-se necessário minimizar o efeito dessa interação, por meio da seleção de cultivares com melhor estabilidade feno- típica (Ramalho et al., 1993). Trabalhos mais recentes de competição de cultivares realizadas na Região Nordeste do Brasil têm procurado recomendar híbridos e cultivares, divulgando materiais que associam boa adaptação nos diferentes ambientes a alta estabilidade de produção (Cardoso et al., 1997; Monteiro et al., 1998; Carvalho et al., 1999a, 1999c, 1999d).

Este trabalho teve o objetivo de conhecer a adaptabilidade e a estabilidade de cultivares de milho em vários locais do Nordeste brasileiro, para dotar a agricultura regional de híbridos e cultivares superiores.

\section{Material e Métodos}

Os ensaios de híbridos e os que envolveram cultivares e populações foram realizados em 19 e 16 ambientes do Nordeste brasileiro, respectivamente, no ano agrícola de 1998, nos Estados do Piauí, Rio Grande do Norte, Pernambuco, Alagoas, Sergipe e Bahia, em diferentes tipos de solo, entre as latitudes $2^{\circ} 63^{\prime} \mathrm{S}$ a $12^{\circ} 41^{\prime} \mathrm{S}$ (Tabela 1). As precipitações pluviais registradas no decorrer do período experimental constam na Tabela 2 .

Os ensaios com cultivares e populações envolveram 25 materiais, ressaltando-se que o termo cultivar é específico para populações em equilíbrio, de polinização livre, enquanto que o termo população refere-se a materiais com as mesmas características, porém em fase de melhoramento. Os ensaios com híbridos contemplaram 30 materiais. Em ambos os casos, utilizou-se o delineamento experimental em blocos ao acaso, em três repetições. Cada parcela constou de quatro fileiras de 5,0 m de comprimento, a espaços de $0,90 \mathrm{~m}$ e 0,50 $\mathrm{m}$ entre covas dentro das fileiras. Foram colocadas três sementes por cova, deixando-se duas plantas por cova, após o desbaste. As adubações de cada experimento foram realizadas de acordo com as análises de solo. Foram colhidas duas fileiras centrais de forma integral, e os pesos de grãos de todos os tratamentos foram ajustados para o nível de $15 \%$ de umidade.

Os dados de produtividade foram submetidos a uma análise de variância por local, obedecendo-se ao modelo em blocos ao acaso, e a uma análise de variância conjunta, obedecendo ao critério de homogeneidade dos quadrados médios residuais, considerando aleatórios os efeitos de blocos e ambientes, e fixo, o efeito de cultivares.

Os parâmetros de adaptabilidade e estabilidade foram estimados segundo a metodologia proposta por Cruz et al. (1989), que utiliza o seguinte modelo:

$Y_{i j}=\beta_{o i}+\beta_{1 i} I_{j}+\beta_{2 i} T\left(I_{j}\right)+\delta_{i j}+\bar{\varepsilon}_{i j}$, em que: $Y_{i j}$ : média da cultivar $\mathrm{i}$ no ambiente $\mathrm{j} ; \mathrm{I}_{\mathrm{j}}$ : índice ambiental; $\mathrm{T}\left(\mathrm{I}_{\mathrm{j}}\right)=0$ 
Tabela 1. Coordenadas geográficas e tipos de solos das áreas experimentais em diversos locais, na Região Nordeste do Brasil, 1998.

\begin{tabular}{|c|c|c|c|c|c|}
\hline Estado & Município & Latitude (S) & Longitude (W) & Altitude (m) & Tipo de solo ${ }^{(1)}$ \\
\hline \multirow[t]{6}{*}{ Piauí } & Teresina (Latossolo) & $5^{\circ} 5^{\prime}$ & $42^{\circ} 49^{\prime}$ & 72 & A \\
\hline & Teresina (Aluvial) & $5^{\circ} 5^{\prime}$ & $42^{\circ} 49^{\prime}$ & 72 & LVA \\
\hline & Angical do Piauí & $6^{\circ} 15^{\prime}$ & $42^{\circ} 55^{\prime}$ & 15 & $\mathrm{BE}$ \\
\hline & Guadalupe & $6^{\circ} 56^{\prime}$ & $43^{\circ} 50^{\prime}$ & 180 & LVA \\
\hline & Parnaíba & $2^{\circ} 63^{\prime}$ & $41^{\circ} 41^{\prime}$ & 15 & AQ \\
\hline & Floriano & $6^{\circ} 46^{\prime}$ & $43^{\circ} 1^{\prime}$ & 85 & A \\
\hline \multirow[t]{2}{*}{ R.G.Norte } & Ipanguassu & $5^{\circ} 37^{\prime}$ & $36^{\circ} 50^{\prime}$ & 70 & A \\
\hline & Cruzeta & $-(2)$ & - & - & - \\
\hline \multirow[t]{2}{*}{ Pernambuco } & Vitória $\mathrm{St}^{\mathrm{o}}$ Antão & $8^{\circ} 12^{\prime}$ & $35^{\circ} 21^{\prime}$ & 350 & LVA \\
\hline & Itambé & $7^{\circ} 21^{\prime}$ & $35^{\circ} 7^{\prime}$ & 190 & LVA \\
\hline Alagoas & União dos Palmares & $9^{\circ} 6^{\prime}$ & $36^{\circ} 4^{\prime}$ & 156 & LVA \\
\hline \multirow[t]{3}{*}{ Sergipe } & N.Sr ${ }^{\mathrm{a}}$ das Dores & $10^{\circ} 30^{\prime}$ & $37^{\circ} 13^{\prime}$ & 200 & LVA \\
\hline & Neópolis & $10^{\circ} 16^{\prime}$ & $36^{\circ} 51^{\prime}$ & 7 & A \\
\hline & Umbaúba & $12^{\circ} 22^{\prime}$ & $37^{\circ} 40^{\prime}$ & 109 & LVA \\
\hline \multirow[t]{5}{*}{ Bahia } & Adustina 1 & $10^{\circ} 32^{\prime}$ & $38^{\circ} 7^{\prime}$ & 250 & LVA \\
\hline & Adustina 2 & $10^{\circ} 32^{\prime}$ & $38^{\circ} 7^{\prime}$ & 250 & PVA \\
\hline & Paripiranga & - & - & - & LVA \\
\hline & Barreiras (Faz. Melancias) & $12^{\circ} 12^{\prime}$ & $46^{\circ} 7^{\prime}$ & 810 & AQ \\
\hline & Barreiras (Faz. St ${ }^{\mathrm{a}}$ Cruz) & $12^{\circ} 14^{\prime}$ & $45^{\circ} 20^{\prime}$ & 670 & AQ \\
\hline
\end{tabular}

(1) A: Aluvial; LVA: Latossolo Vermelho-Amarelo; PVA: Podzólico Vermelho-Amarelo; BE: Brunizém-Escuro; AQ: Areia Quartzosa. (2)Dados não encontrados na fonte consultada.

Tabela 2. Índices pluviométricos ( $\mathrm{mm}$ ) ocorridos durante o período experimental, em diferentes locais da Região Nordeste do Brasil, 1998.

\begin{tabular}{|c|c|c|c|c|c|c|c|c|c|}
\hline Local & Janeiro & Fevereiro & Março & Abril & Maio & Junho & Julho & Agosto & Total \\
\hline Teresina (Latossolo) & 287,5 & 143,9 & 210,9 & 89,4 & 10,4 & $-(1)$ & - & - & 741,7 \\
\hline Teresina (Aluvial) & 287,5 & 143,9 & 210,9 & 89,4 & 10,4 & - & - & - & 741,7 \\
\hline Angical do Piauí & 159,0 & 189,2 & 248,0 & 73,4 & 8,4 & - & - & - & 678,0 \\
\hline Guadalupe & 254,6 & 117,9 & 131,8 & 3,1 & 3,0 & - & - & - & 538,4 \\
\hline Parnaíba & 193,6 & 30,5 & 182,5 & 51,3 & 103,2 & - & - & - & 60,1 \\
\hline Floriano & - & 181,3 & 180,0 & 86,0 & 9,5 & - & - & - & 604,4 \\
\hline Vitória $\mathrm{St}^{\circ}$ Antão & - & - & - & 46,1 & 102,8 & 33,2 & 65,5 & 95,9 & 343,5 \\
\hline Itambé & - & - & - & 60,6 & 133,6 & 52,0 & 157,0 & 141,6 & 545,8 \\
\hline União Palmares & - & - & - & - & - & - & - & - & - \\
\hline N. $\mathrm{Sr}^{\mathrm{a}}$ das Dores & - & - & - & - & 120,0 & 219,0 & 154,0 & 60,0 & 553,0 \\
\hline Neópolis & - & - & - & - & 198,0 & 223,0 & 301,0 & 83,0 & 805,0 \\
\hline Umbaúba & - & - & - & 112,0 & 268,0 & 425,0 & 269,0 & 135,0 & $1.309,0$ \\
\hline Adustina 1 & - & - & - & 70,0 & 82,0 & 160,0 & 200,0 & 82,0 & 594,0 \\
\hline Adustina 2 & - & - & - & 104,0 & 111,0 & 250,0 & 213,0 & 125,0 & 903,0 \\
\hline Paripiranga & - & - & - & 104,0 & 111,0 & 250,0 & 213,0 & 125,0 & 903,0 \\
\hline Barreiras (Faz. Melancias) & 570,0 & 194,0 & 91,0 & 42,0 & - & - & - & - & 584,0 \\
\hline Barreiras (Faz. St ${ }^{\mathrm{a}}$ Cruz) & 150,0 & 121,0 & 29,0 & 14,0 & - & - & - & - & 314,0 \\
\hline Ipanguassu & - & - & - & - & - & - & - & - & - \\
\hline Cruzeta & - & - & - & - & - & - & - & - & - \\
\hline
\end{tabular}

(1) Fora do período experimental ou dados não registrados. 
se $\mathrm{I}_{\mathrm{j}}<0 ; \mathrm{T}\left(\mathrm{I}_{\mathrm{j}}\right)=\left(\mathrm{I}_{\mathrm{j}}\right)-\overline{\mathrm{I}}$ se $\mathrm{I}_{\mathrm{j}}>0$, sendo $\mathrm{I}_{+}$a média dos índices positivos; $\beta_{\mathrm{oi}}$ : média geral da cultivar $\mathrm{i} ; \beta_{1 \mathrm{j}}$ : coeficiente de regressão linear associado à variável $\mathrm{I}_{\mathrm{j}} ; \beta_{2 \mathrm{i}}$ : coeficiente da regressão linear associado à variável $\mathrm{T}\left(\mathrm{I}_{\mathrm{j}}\right)$; $\delta_{\mathrm{ij}}$ : desvio da regressão linear; $\bar{\varepsilon}_{\mathrm{ij}}$ : erro experimental médio.

\section{Resultados e Discussão}

As produtividades médias de grãos nos ensaios de cultivares e populações variaram de $1.864 \mathrm{~kg} / \mathrm{ha}$ (Barreiras/Fazenda Santa Cruz) a $5.321 \mathrm{~kg} / \mathrm{ha}$ (Adustina 1), indicando uma ampla faixa de variação nas condições ambientais em que foram realizados (Tabela 3). Os municípios Teresina (em solo do tipo Aluvial), no Piauí, Vitória de Santo Antão, em Pernambuco, Nossa Senhora das Dores e Neópolis, em Sergipe e Paripiranga e Adustina 1, na Bahia, apresentaram maiores potencialidades para o desenvolvimento do milho, com produtividades médias entre $5.099 \mathrm{~kg} / \mathrm{ha}$ a $5.321 \mathrm{~kg} / \mathrm{ha}$. Vale ressaltar que os rendimentos médios obtidos nesses ambientes colocam essas áreas em condições de competir com a exploração do milho em áreas de cerrados, cuja produtividade ultrapassa os $5.000 \mathrm{~kg} / \mathrm{ha}$. Nesses ensaios, os coeficientes de variação obtidos oscilaram entre $6,6 \%$ a $21,7 \%$, conferindo boa precisão aos trabalhos, segundo critérios adotados por Scapim et al. (1995).

Nos ensaios de híbridos, a média geral registrada foi de $4.884 \mathrm{~kg} / \mathrm{ha}$, observando-se uma variação entre os ambientes de $2.189 \mathrm{~kg} / \mathrm{ha}$ (Ibititá) a $6.743 \mathrm{~kg} / \mathrm{ha}$ (Teresina, em solo do tipo Aluvial), destacando-se como mais propícios ao cultivo de híbridos os municípios de Floriano, Parnaíba e Teresina, no Piauí, Ipanguassu, no Rio Grande do Norte, Nossa Senhora das Dores e Neópolis, em Sergipe, e Adustina 2, na Bahia, com rendimentos médios variando de $5.277 \mathrm{~kg} / \mathrm{ha}$ a $6.743 \mathrm{~kg} / \mathrm{ha}$ (Tabela 3). Estes resultados também colocam essas áreas em condições de competir com áreas do cerrado, na exploração de híbridos de milho. Os coeficientes de variação obtidos indicam boa precisão desses ensaios, de acordo com a classificação de Scapim et al. (1995).

Os híbridos mostraram superioridade, nos seus rendimentos médios, de $21,4 \%$ em relação às cultivares e populações, evidenciando melhor adaptação às condições edafoclimáticas da região. Resultados semelhantes têm sido constatados em outros traba- lhos na região (Cardoso et al., 1997; Monteiro et al., 1998; Carvalho et al., 1999d).

A análise de variância conjunta, tanto dos ensaios envolvendo cultivares e populações quanto dos ensaios com híbridos, evidenciou efeitos significativos a $1 \%$ de probabilidade, pelo teste $\mathrm{F}$, para ambientes, tratamentos e interação ambientes x tratamentos. Constatada a presença da interação, foram estimados os parâmetros de adaptabilidade e estabilidade, propostos por Cruz et al. (1989). Aliado ao modelo proposto, consideram-se como cultivares melhor adaptadas as que expressaram maiores produtividades de grãos (Mariotti et al., 1976).

A produtividade média de grãos dos ensaios envolvendo cultivares e populações variou de $2.878 \mathrm{~kg} / \mathrm{ha}$ (CMS 47) a $5.504 \mathrm{~kg} /$ ha (Cargill 909), com média geral de $4.022 \mathrm{~kg} / \mathrm{ha}$, o que expressa o potencial desses materiais na região (Tabela 4). Os híbridos Cargill 909 e BR 3123, utilizados como testemunhas, apresentaram os melhores rendimentos. As cultivares AL 30 e BR 5028-São Francisco destacaram-se, juntamente com o Sintético Dentado e CMS 50, como as mais produtivas entre as cultivares e populações. Analisando-se o comportamento das cultivares dotadas de produtividade média acima da média geral, a estimativa de $\beta_{1}$ que avalia seus desempenhos nas condições desfavoráveis indicou os híbridos Cargill 909 e BR 2121 e as cultivares BR 5028-São Francisco e BR 5033-Asa Branca, como responsivos a essas condições $\left(\beta_{1}>1\right)$. A estimativa de $\beta_{1}+\beta_{2}$, que avalia a resposta das cultivares nos ambientes favoráveis, evidenciou os híbridos Cargill 909 e BR 3123 e as cultivares AL 30, Sintético Dentado, Sintético Duro e BR 5033-Asa Branca como responsivos à melhoria ambiental $\left(\beta_{1}+\beta_{2}>1\right)$.

A maioria das cultivares e populações avaliadas mostrou os desvios de regressão estatisticamente diferentes de zero, o que, pelo método proposto, indica comportamento imprevisível nos ambientes considerados (Tabela 4). Apesar disso, Cruz et al. (1989), consideram que materiais que apresentem valores de $\mathrm{R}^{2}>\mathbf{8 0 \%}$ não devem ter o seu grau de previsibilidade comprometido. Dessa forma, a estimativa de $\mathrm{R}^{2}$ para as cultivares de melhor adaptação, consideradas as dotadas de produtividade média acima da média geral, à exceção do híbrido BR 3123 (testemunha), foi superior a $80 \%$, o que evidencia bom ajustamento às retas de regressão do modelo. 
Tabela 3. Produtividades médias de grãos e coeficientes de variação obtidos nos ensaios de cultivares e de híbridos, em diferentes locais (ambientes) da Região Nordeste do Brasil, 1998.

\begin{tabular}{|c|c|c|c|c|}
\hline \multirow[t]{2}{*}{ Ambiente } & \multicolumn{2}{|c|}{ Variedades e populações } & \multicolumn{2}{|c|}{ Híbridos } \\
\hline & $\begin{array}{l}\text { Produtividade } \\
\text { média }(\mathrm{kg} / \mathrm{ha})\end{array}$ & $\begin{array}{c}\text { Coeficiente de } \\
\text { variação }(\%)\end{array}$ & $\begin{array}{l}\text { Produtividade } \\
\text { média }(\mathrm{kg} / \mathrm{ha})\end{array}$ & $\begin{array}{c}\text { Coeficiente de } \\
\text { variação (\%) }\end{array}$ \\
\hline \multicolumn{5}{|l|}{ Piauí } \\
\hline Floriano & 4.381 & 8,8 & 5.414 & 8,1 \\
\hline Parnaíba & 4.473 & 7,7 & 5.277 & 6,0 \\
\hline Teresina (Latossolo) & 4.672 & 6,9 & 4.851 & 7,1 \\
\hline Teresina (Aluvial) & 5.099 & 6,6 & 6.743 & 5,8 \\
\hline Angical do Piauí & 3.869 & 9,6 & 4.918 & 6,4 \\
\hline Guadalupe & 2.597 & 15,7 & 3.294 & 14,9 \\
\hline \multicolumn{5}{|l|}{ Rio Grande do Norte } \\
\hline Cruzeta & 3.170 & 13,3 & $-(1)$ & - \\
\hline Ipanguassu & 4.061 & 11,7 & 5.134 & 10,2 \\
\hline \multicolumn{5}{|l|}{ Pernambuco } \\
\hline Itambé & 2.826 & 15,7 & 3.904 & 11,5 \\
\hline Vitória de Santo Antão & 5.236 & 12,1 & - & - \\
\hline \multicolumn{5}{|l|}{ Alagoas } \\
\hline União dos Palmares & 3.553 & 14,5 & - & - \\
\hline \multicolumn{5}{|l|}{ Sergipe } \\
\hline Nossa Senhora das Dores & 5.130 & 11,6 & 6.665 & 10,0 \\
\hline Neópolis & 5.237 & 8,3 & 6.465 & 10,8 \\
\hline Umbaúba & 3.446 & 13,0 & 4.487 & 11,8 \\
\hline \multicolumn{5}{|l|}{ Bahia } \\
\hline Adustina 1 & 5.321 & 9,3 & 4.257 & 14,7 \\
\hline Adustina 2 & 2.681 & 21,7 & 5.824 & 9,5 \\
\hline Paripiranga & 5.282 & 12,9 & - & - \\
\hline Barreiras (Faz. Melancias) & 3.509 & 12,4 & 4.828 & 9,0 \\
\hline Barreiras (Faz. Santa Cruz) & 1.864 & 19,9 & 3.130 & 14,4 \\
\hline Ibititá & - & - & 2.189 & 19,3 \\
\hline Média & 4.022 & & 4.884 & \\
\hline
\end{tabular}

(1)Não houve ensaio.

A cultivar ideal preconizada pelo modelo bissegmentado (média alta, $\beta_{1}<1, \beta_{1}+\beta_{2}>1$ e desvio da regressão igual a zero) não foi encontrada no conjunto avaliado (Tabela 4). Da mesma forma, não foram identificadas cultivares com adaptação específica a ambientes desfavoráveis (média alta, $\beta_{1}<1 \mathrm{e}$ $\left.\beta_{1}+\beta_{2}<1\right)$. Entretanto, pelas posições de suas médias nesses ambientes, destacou-se o híbrido Cargill 909, que, embora responsivo a ambientes desfavoráveis $\left(\beta_{1}>1\right)$, apresentou bom desempenho, com produtividade média superior à de todas as demais. As cultivares BR 5028, também com $\beta_{1}>1$, e AL 30, Sintético Dentado, Sintético Duro e BR 106, $\operatorname{com} \beta_{1}=1$, também mostraram bom desempenho. O híbrido BR 3123, apesar de apresentar $\mathrm{R}^{2}<80 \%$, foi o segundo material mais produtivo nos ambientes desfavoráveis.

Nos ambientes favoráveis, merece destaque o híbrido Cargill 909, por apresentar média alta, ser exigente nas condições desfavoráveis e responsivo à melhoria ambiental $\left(\beta_{1}>1\right.$ e $\left.\beta_{1}+\beta_{2}>1\right)$. A cultivar BR 5033-Asa Branca, de produtividade média pouco superior em relação à média geral, também atende aos requisitos para recomendação nesses ambientes. O híbrido BR 3123 e as cultivares AL 30, Sintético Dentado e Sintético Duro, introduzidas na re- 
Tabela 4. Produtividade média de grãos (kg/ha) e estimativa dos parâmetros de adaptabilidade e estabilidade de 25 cultivares de milho em 19 ambientes da Região Nordeste do Brasil, 1998

\begin{tabular}{|c|c|c|c|c|c|c|c|c|}
\hline \multirow[t]{2}{*}{ Cultivar } & \multicolumn{3}{|c|}{ Média nos ambientes } & \multirow[t]{2}{*}{$\hat{\beta}_{1}$} & \multirow[t]{2}{*}{$\hat{\beta}_{2}$} & \multirow[t]{2}{*}{$\hat{\beta}_{1}+\hat{\beta}_{2}$} & \multirow[t]{2}{*}{ S } & \multirow[t]{2}{*}{$\mathrm{R}^{2}$} \\
\hline & Geral & Desfavorável & Favorável & & & & & \\
\hline Cargill 909 & 5.504 & 4.248 & 6.634 & $1,37 * *$ & $1,08 * *$ & $2,46^{* *}$ & $1.345,40^{* * *}$ & 83 \\
\hline BR 3123 & 5.002 & 4.141 & 5.780 & $0,98^{\mathrm{ns}}$ & $0,50 *$ & $1,49^{*}$ & $1.200,06^{* *}$ & 75 \\
\hline AL 30 & 4.772 & 3.526 & 5.893 & $0,94^{\mathrm{ns}}$ & $0,56 * *$ & $1,94 * *$ & $1.085,30 * *$ & 87 \\
\hline BR 5028 & 4.505 & 3.566 & 5.350 & $1,38 * *$ & $0,16^{\mathrm{ns}}$ & $1,09^{\mathrm{ns}}$ & $775,40 * *$ & 85 \\
\hline BR 2121 & 4.389 & 3.174 & 5.483 & $1,31 * *$ & $-0,20^{\mathrm{ns}}$ & $1,10^{\mathrm{ns}}$ & $780,01 * *$ & 91 \\
\hline Sintético Dentado & 4.262 & 3.428 & 5.013 & $0,94^{\mathrm{ns}}$ & $1,17 * *$ & $2,11 * *$ & $1.050,18^{* *}$ & 81 \\
\hline CMS 50 & 4.208 & 3.153 & 5.156 & $1,06^{\mathrm{ns}}$ & $0,19^{\mathrm{ns}}$ & $1,26^{\mathrm{ns}}$ & $961,02 * *$ & 83 \\
\hline Sintético Duro & 4.198 & 3.231 & 5.068 & $1,02^{\mathrm{ns}}$ & $0,46^{*}$ & $1,48^{*}$ & $707,96^{* *}$ & 89 \\
\hline BR 106 & 4.142 & 3.274 & 4.923 & $0,97^{\mathrm{ns}}$ & $0,11^{\mathrm{ns}}$ & $1,09^{\mathrm{ns}}$ & $918,34 * *$ & 82 \\
\hline BR 5011 & 4.125 & 3.067 & 5.078 & $0,99^{\mathrm{ns}}$ & $0,03^{\mathrm{ns}}$ & $1,02^{\mathrm{ns}}$ & $750,63 * *$ & 87 \\
\hline BR 5033 & 4.124 & 2.973 & 5.161 & $1,15^{*}$ & $0,26^{\mathrm{ns}}$ & $1,42 *$ & $506,26^{\mathrm{ns}}$ & 96 \\
\hline CMS 453 & 4.077 & 3.035 & 5.016 & $0,98^{\mathrm{ns}}$ & $0,05^{\mathrm{ns}}$ & $1,04^{\mathrm{ns}}$ & $628,91^{\mathrm{ns}}$ & 91 \\
\hline CMS 59 & 3.999 & 2.976 & 4.920 & $0,99^{\mathrm{ns}}$ & $0,01^{\mathrm{ns}}$ & $1,01^{\mathrm{ns}}$ & $930,07 * *$ & 82 \\
\hline BR 5039 & 3.970 & 2.713 & 5.101 & $1,19 *$ & $-0,66^{* *}$ & $0,52^{*}$ & $2.828,29 * *$ & 87 \\
\hline BR 5037 & 3.951 & 3.055 & 4.756 & $0,93^{\mathrm{ns}}$ & $0,19^{\mathrm{ns}}$ & $1,13^{\mathrm{ns}}$ & $1.070,68 * *$ & 76 \\
\hline AL 25 & 3.923 & 2.993 & 4.760 & $0,98^{\mathrm{ns}}$ & $0,06^{\mathrm{ns}}$ & $1,05^{\mathrm{ns}}$ & $364,62^{\mathrm{ns}}$ & 97 \\
\hline Across 8528 & 3.846 & 2.929 & 4.670 & $0,95^{\mathrm{ns}}$ & $0,06^{\mathrm{ns}}$ & $1,02^{\mathrm{ns}}$ & $789,44 * *$ & 85 \\
\hline CMS 22 & 3.842 & 2.857 & 4.729 & $0,93^{\mathrm{ns}}$ & $-0,76^{* *}$ & $0,16^{* *}$ & $943,59 * *$ & 77 \\
\hline Pool 18 & 3.761 & 2.958 & 4.484 & $0,86^{*}$ & $0,10^{\mathrm{ns}}$ & $0,96^{\mathrm{ns}}$ & $921,55^{* *}$ & 78 \\
\hline CMS 52 & 3.605 & 2.698 & 4.422 & $0,91^{\mathrm{ns}}$ & $-0,61 * *$ & $0,29 * *$ & $607,79^{\mathrm{ns}}$ & 89 \\
\hline CMS 35 & 3.426 & 2.785 & 4.003 & $0,75 * *$ & $-0,06^{\mathrm{ns}}$ & $0,68^{\mathrm{ns}}$ & $884,18^{* *}$ & 74 \\
\hline BR 473 & 3.356 & 2.497 & 4.129 & $0,91^{\mathrm{ns}}$ & $-0,59 * *$ & $0,31 * *$ & $669,45^{*}$ & 87 \\
\hline Saracura & 3.327 & 2.325 & 4.230 & $0,94^{\mathrm{ns}}$ & $-0,50 * *$ & $0,43 * *$ & $889,42 * *$ & 80 \\
\hline BR 5004 & 3.325 & 2.401 & 4.157 & $0,96^{\mathrm{ns}}$ & $-1,10 * *$ & $-0,14 * *$ & $1.368,32 * *$ & 64 \\
\hline CMS 47 & 2.878 & 2.459 & 3.256 & $0,51 *$ & $-0,55 * *$ & $-0,03 * *$ & 854,73 ** & 57 \\
\hline Média & 4.022 & & & & & & & \\
\hline
\end{tabular}

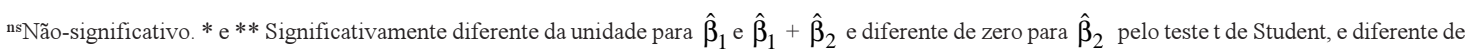
zero para o quadrado médio dos desvios de regressão (S), pelo teste $\mathrm{F}$, a $5 \%$ e $1 \%$ de probabilidade, respectivamente.

gião no ano agrícola de 1998, mostraram boa adaptação (média alta) nos ambientes favoráveis, com respostas positivas à melhoria ambiental $\left(\beta_{1}+\beta_{2}>1\right)$. O híbrido BR 2121 e a cultivar BR 5028-São Francisco, embora não tenham apresentado resposta à melhoria ambiental $\left(\beta_{1}+\beta_{2}=1\right)$, situaram-se entre os mais produtivos nesses ambientes. A população CMS 50 e as cultivares BR 106 e BR 5011-Sertanejo, de ampla adaptabilidade nos ambientes estuda$\operatorname{dos}\left(\beta_{1}=1\right.$ e $\left.\beta_{1}+\beta_{2}=1\right)$, também apresentaram rendimentos que justificam sua recomendação para a região. Todas essas cultivares, à exceção das AL 30, Sintético Dentado e Sintético Duro, vêm repetindo bom comportamento na região, conforme tem sido assinalado por Cardoso et al. (1997), Carvalho et al. (1999a, 1999c, 1999d).

Os parâmetros de adaptabilidade e estabilidade estimados com relação aos híbridos constam na Tabela 5. A produtividade média de grãos obtida em dezesseis ambientes foi de $4.884 \mathrm{~kg} / \mathrm{ha}$, com variação de $3.791 \mathrm{~kg} / \mathrm{ha}$ (Planagri 8440) a $5.616 \mathrm{~kg} / \mathrm{ha}$ (Dina 1000), constatando-se que os híbridos melhor adaptados à Região Nordeste foram o Dina 1000, Cargill 333 B, Dina 270, Braskalb XL 345, Pioneer 3021, Master, dentre outros. Entre os menos adaptados, podem ser citados os Planagri 8440, AG 5011, BR 2121, AG 9012, AG 9014 e Agromen 3100. Verificou-se que, dentre os mais produtivos, os híbridos Pioneer 3021 e Zeneca 8452 foram os menos exigentes nos ambientes desfavoráveis $\left(\beta_{1}<1\right)$, enquanto que os AG 1051, AG 4051, Cargill 909, Pioneer X 1296 B e Cargill 901 mostraram-se exigentes nessa condição $\left(\beta_{1}>1\right)$. Os híbridos Cargill 333 B, Pioneer 3021, Master, AG 4051, Pioneer 3027, Pioneer X 1296 B e Zeneca 8501 foram os mais responsivos à melhoria ambiental $\left(\beta_{1}+\beta_{2}>1\right)$. 
Tabela 5. Produtividade média de grãos (kg/ha) e estimativa dos parâmetros de adaptabilidade e estabilidade de 30 híbridos de milho em 16 ambientes da Região Nordeste do Brasil, 1998.

\begin{tabular}{|c|c|c|c|c|c|c|c|c|}
\hline \multirow[t]{2}{*}{ Híbrido } & \multicolumn{3}{|c|}{ Médias nos ambientes } & \multirow{2}{*}{$\hat{\beta}_{1}$} & \multirow{2}{*}{$\hat{\beta}_{2}$} & \multirow{2}{*}{$\hat{\beta}_{1}+\hat{\beta}_{2}$} & \multirow[t]{2}{*}{ S } & \multirow[t]{2}{*}{$\mathrm{R}^{2}$} \\
\hline & Geral & Desfavorável & Favorável & & & & & \\
\hline Dina $1000^{(1)}$ & 5.616 & 4.753 & 6.485 & $1,00^{\mathrm{ns}}$ & $0,22^{\mathrm{ns}}$ & $1,22^{\mathrm{ns}}$ & $1.325 .510,12^{* *}$ & 83 \\
\hline Cargill $333 \mathrm{~B}^{(2)}$ & 5.598 & 4.655 & 6.541 & $0,94^{\text {ns }}$ & $0,42^{* *}$ & $1,36^{*}$ & $971.248,62^{* *}$ & 86 \\
\hline Dina $270^{(1)}$ & 5.453 & 4.384 & 6.522 & $1,10^{\mathrm{ns}}$ & $-0,24^{\mathrm{ns}}$ & $0,85^{\text {ns }}$ & $1.216 .442,50^{* *}$ & 85 \\
\hline Braskalb XL $360^{(2)}$ & 5.453 & 4.563 & 6.342 & $0,91^{\mathrm{ns}}$ & $0,14^{\mathrm{ns}}$ & $1,06^{\text {ns }}$ & $359.104,00^{\text {ns }}$ & 94 \\
\hline Pioneer $3021^{(2)}$ & 5.438 & 4.717 & 6.152 & $0,86^{*}$ & $0,64^{* *}$ & $1,51^{* *}$ & $2.674 .486,25^{* *}$ & 68 \\
\hline Master $^{(2)}$ & 5.438 & 4.401 & 6.476 & $0,97^{\mathrm{ns}}$ & $0,45^{* *}$ & $1,43^{* *}$ & $1.009 .835,06^{* *}$ & 87 \\
\hline AG $1051^{(3)}$ & 5.374 & 4.112 & 6.635 & $1,37^{* *}$ & $-0,61^{* *}$ & $0,75^{\text {ns }}$ & $2.257 .078,75^{* *}$ & 81 \\
\hline AG $4051^{(2)}$ & 5.355 & 4.195 & 6.514 & $1,20^{* *}$ & $0,08^{\mathrm{ns}}$ & $1,29^{*}$ & $685.186,43^{* *}$ & 93 \\
\hline Pioneer $3041^{(2)}$ & 5.297 & 4.141 & 6.453 & $1,18^{* *}$ & $0,01^{\mathrm{ns}}$ & $1,20^{\mathrm{ns}}$ & $806.152,62^{* *}$ & 91 \\
\hline Cargill $909^{(1)}$ & 5.279 & 4.211 & 6.347 & $1,22^{* *}$ & $0,01^{\text {ns }}$ & $1,22^{\text {ns }}$ & $2.648 .027,00^{* * *}$ & 77 \\
\hline Braskalb XL $345^{(2)}$ & 5.257 & 4.186 & 6.327 & $1,08^{\mathrm{ns}}$ & $-0,02^{\mathrm{ns}}$ & $1,06^{\mathrm{ns}}$ & $370.052,92^{\text {ns }}$ & 95 \\
\hline Pioneer $3027^{(2)}$ & 5.176 & 4.288 & 6.064 & $0,99^{\mathrm{ns}}$ & $0,35^{\mathrm{ns}}$ & $1,34^{*}$ & $855.454,74^{* *}$ & 88 \\
\hline Pioneer X 1296 B & 5.118 & 3.865 & 6.372 & $1,18^{* *}$ & $0,11^{\text {ns }}$ & $1,29^{*}$ & $1.046 .344,62^{* *}$ & 89 \\
\hline Cargill 901 ${ }^{(1)}$ & 4.999 & 3.886 & 6.112 & $1,20^{* *}$ & $-0,33^{*}$ & $0,86^{\mathrm{ns}}$ & $1.127 .731,12^{* *}$ & 87 \\
\hline Zeneca $8452^{(1)}$ & 4.997 & 4.197 & 5.796 & $0,84^{*}$ & $0,44^{* *}$ & $1,29^{*}$ & $1.840 .167,37^{* *}$ & 73 \\
\hline Zeneca $8501^{(2)}$ & 4.919 & 3.937 & 5.901 & $0,98^{\mathrm{ns}}$ & $0,62^{* *}$ & $1,61^{* *}$ & $1.299 .681,87^{* *}$ & 84 \\
\hline Colorado $32^{(2)}$ & 4.914 & 4.130 & 5.698 & $0,91^{\mathrm{ns}}$ & $0,14^{\mathrm{ns}}$ & $1,06^{\mathrm{ns}}$ & $1.228 .808,62^{* *}$ & 81 \\
\hline $\operatorname{Ag} 5014^{(2)}$ & 4.680 & 3.629 & 5.730 & $1,06^{\mathrm{ns}}$ & $-0,20^{\mathrm{ns}}$ & $0,85^{\text {ns }}$ & $302.070,15^{\text {ns }}$ & 95 \\
\hline Colorado $42^{(3)}$ & 4.623 & 3.730 & 5.515 & $0,94^{\mathrm{ns}}$ & $0,44^{* *}$ & $1,38^{* *}$ & $580.991,37^{* *}$ & 91 \\
\hline Cargill $435^{(3)}$ & 4.598 & 3.695 & 5.501 & $0,89^{\text {ns }}$ & $-0,01^{\mathrm{ns}}$ & $0,87^{\text {ns }}$ & $580.991,37^{* *}$ & 86 \\
\hline Agromen $2003^{(3)}$ & 4.587 & 3.538 & 5.635 & $1,07^{\mathrm{ns}}$ & $0,35^{*}$ & $1,42^{* *}$ & $532.665,87^{* *}$ & 93 \\
\hline AG $3010^{(3)}$ & 4.575 & 3.844 & 5.304 & $0,76^{* *}$ & $0,50^{* *}$ & $1,26^{*}$ & $742.570,75^{* *}$ & 85 \\
\hline MR $2601^{(1)}$ & 4.556 & 3.501 & 5.610 & $1,02^{\mathrm{ns}}$ & $-0,53^{* *}$ & $0,49^{* *}$ & $1.394 .880,00^{* *}$ & 80 \\
\hline BR $3123^{(2)}$ & 4.515 & 3.731 & 5.299 & $0,76^{* *}$ & $-0,21^{\mathrm{ns}}$ & $0,54^{* *}$ & $927.757,25^{* *}$ & 77 \\
\hline Agromen $3100^{(3)}$ & 4.303 & 3.314 & 5.292 & $1,00^{\text {ns }}$ & $-0,01^{\text {ns }}$ & $0,89^{\mathrm{ns}}$ & $1.086 .080,62^{* *}$ & 84 \\
\hline AG $9014^{(1)}$ & 4.234 & 3.128 & 5.340 & $0,89^{\text {ns }}$ & $-0,86^{* *}$ & $0,03^{* *}$ & $3.673 .981,50^{* * *}$ & 52 \\
\hline AG $9012^{(1)}$ & 4.220 & 3.152 & 5.287 & $1,02^{\mathrm{ns}}$ & $-0,94^{* *}$ & $0,07^{* *}$ & $2.019 .387,75^{* *}$ & 72 \\
\hline BR $2121^{(3)}$ & 4.206 & 3.510 & 4.901 & $0,73^{* *}$ & $0,30^{\text {ns }}$ & $1,04^{\mathrm{ns}}$ & $302.908,00^{\text {ns }}$ & 92 \\
\hline AG $5011^{(2)}$ & 3.943 & 2.787 & 5.098 & $1,13^{*}$ & $-0,92^{* *}$ & $0,20^{* *}$ & $699.164,00^{* *}$ & 77 \\
\hline Planagri $8440^{(3)}$ & 3.791 & 3.126 & 4.460 & $0,65^{* *}$ & $-0,25^{\mathrm{ns}}$ & $0,40^{* *}$ & $2.452 .360,00^{* *}$ & 72 \\
\hline
\end{tabular}

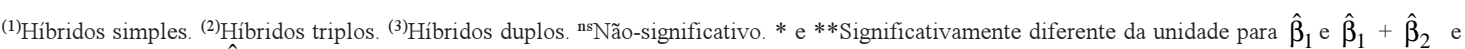
diferente de zero para $\hat{\beta}_{2}$ pelo teste t de Student, e diferente de zero para o quadrado médio dos desvios de regressão (S), pelo teste F, a $5 \%$ e $1 \%$ de probabilidade, respectivamente.

À exceção dos híbridos Braskalb XL 360, Braskalb XL 345, AG 5014 e BR 2121, todos os outros mostraram desvios da regressão estatisticamente diferente de zero. Entretanto, seguindo o critério de Cruz et al. (1989), dentre os híbridos mais produtivos apenas os Pioneer 3021, Cargill 909 e Zeneca 8452 mostraram baixa previsibilidade, com $\mathrm{R}^{2}<80 \%$.

O genótipo ideal preconizado pelo modelo bissegmentado não foi encontrado no conjunto avaliado, apesar de os híbridos Pioneer 3021 e Zeneca 8452 atenderem ao maior número de requisitos para serem considerados como tais, por apresentarem boa adaptação ( $\beta_{0}$ alto), baixa exigência em ambientes desfavoráveis $(\beta<1)$ e respostas positivas à melhoria ambiental $\left(\beta_{1}+\beta_{2}>1\right)$ (Tabela 5$)$. Como desvantagens mostraram baixa previsibilidade nos ambientes considerados com desvios da regressão diferentes de zero e $\mathrm{R}^{2}<80 \%$.

Neste ensaio não foram identificados híbridos com adaptação específica a ambientes desfavoráveis. Entretanto híbridos com boa previsibilidade e médias altas nesses ambientes, tornam-se de importância para a agricultura regional, a exemplo de Dina 1000, Cargill 333 B, Braskalb XL 360, entre outros. Com adaptação específica a ambientes favoráveis, destacam-se os AG 4051 e Pioneer X 1296 B, os quais expressaram altos rendimentos, exigência nas condições desfavoráveis e resposta favorável à melhoria 
ambiental $\left(\beta_{0}\right.$ alto, $\beta>1$ e $\left.\beta_{1}+\beta_{2}>1\right)$. Os híbridos AG 1051 e Pioneer 3041, ambos exigentes nas condições desfavoráveis, e os Cargill 333 B e Master, responsivos à melhoria ambiental, também apresentaram altas produtividades nos ambientes favoráveis. Os híbridos Dina 1000 e Dina 270, de adaptação geral nos ambientes estudados e altos rendimentos, constituem alternativas importantes para a região.

\section{Conclusões}

1. O híbrido Cargill 909 apresenta alta produtividade, se comparado às cultivares em ambientes favoráveis e desfavoráveis, com boa previsibilidade de produção.

2. As cultivares melhoradas AL 30, BR 5028-São Francisco, BR 106, BR 5011-Sertanejo, BR 5033 Asa Branca, Sintético Dentado, Sintético Duro e a população CMS 50, embora produzam menos que os híbridos, apresentam bons rendimentos e estabilidade de produção e têm importância fundamental nos sistemas de produção dos pequenos e médios produtores rurais da Região Nordeste.

3. Os híbridos Dina 1000, Cargill 333 B, Dina 270, Braskalb XL 360, Master, AG 1051, AG 4051 e Pioneer 3041 apresentam maior adaptação à Região Nordeste com alta previsibilidade de produção nos ambientes considerados.

4. Entre os híbridos mais produtivos, apenas o Pioneer 3021 e Zeneca 8452 mostram pouca exigência nos ambientes desfavoráveis.

5. Os híbridos Dina 1000, Cargill 333 B, Braskalb XL 360, entre outros, apresentam bom desempenho em ambientes desfavoráveis, podendo ser recomendados para esses ambientes.

6. Os híbridos AG 4051 e Pioneer X 1296 B se destacam para recomendação específica a ambientes favoráveis.

\section{Referências}

CARDOSO, M. J.; CARVALHO, H. W. L. de; PACHECO, C. A. P.; SANTOS, M. X. dos; LEAL, M. de L. da S.Adaptabilidade e estabilidade de cultivares de milho no Estado do Piauí no biênio 1993/94. Revista Científica Rural, Bagé, v. 2, n. 1, p. 35-44, 1997

CARVALHO, H. W. L. de; LEAL, M. de L. da S.; SANTOS, M. X. dos; CARDOSO, M. J.; MONTEIRO, A. A. T. Adaptabilidade e estabilidade de variedades e hí- bridos de milho no Nordeste brasileiro. Revista Científica Rural, Bagé, v. 4, n. 1, p. 25-34, 1999a.

CARVALHO, H. W. L. de; SANTOS, M. X. dos; LEAL, M. de L. da S.; CARVALHO, B. C. L. de; LIRA, M. A. Adaptabilidade de cultivares de milho no Nordeste brasileiro no triênio 1994/95/96. Revista Científica Rural, Bagé, v. 4, n. 2, p. 96-104, 1999b.

CARVALHO, H. W. L. de; SANTOS, M. X. dos; LEAL, M. de L. da S.; PACHECO, C. A. P.; CARDOSO, M. J.; MONTEIRO, A. A. T. Adaptabilidade e estabilidade de produção de cultivares de milho no Nordeste brasileiro. Pesquisa Agropecuária Brasileira, Brasília, v. 34, n. 9 , p. 1581-1591, set. $1999 \mathrm{c}$.

CARVALHO, H. W. L. de; SANTOS, M. X. dos; LEAL, M. de L. da S.; PACHECO, C. A. P.; TABOSA, J. N. Adaptabilidade e estabilidade de comportamento e cultivares de milho em treze ambientes nos tabuleiros costeiros do Nordeste brasileiro. Pesquisa Agropecuária Brasileira, Brasília, v. 34, n. 12, p. 2225-2234, dez. 1999d.

CRUZ, D. D.; TORRES, R. T. de; VENCOVSKY, R. Alternative approach to the stability analysis proposed by Silva and Barreto. Revista Brasileira de Genética, v. 12, n. 13, p. $567-582,1989$

MARIOTTI, I. A.; OYARZABAL, E. S.; OSA, J. M.; BULACIO, A. N. R.; ALMADA, G. H. Análisis de estabilidad y adaptabilidad de genotipos de cana de azúcar. I. Interracciones dentro de una localidad experimental. Revista Agronómica del Noroeste Argentino, Tucumán, v. 13, n. 14, p. 105-127, 1976.

MONTEIRO, A. A. T.; CARVAlHO, H. W. L. de; PACHECO, C. A. P.; SANTOS, M. X. dos; ANTERO NETO, J. F.; LEAL, M. de L. da S. Adaptabilidade e estabilidade de cultivares de milho no Estado do Ceará. Revista Científica Rural, Bagé, v. 3, n. 2, p. 1-10, 1998.

RAMALHO, M. A. P.; SANTOS, J. B. dos; ZIMMERMAN, M. J. O. Interação dos genótipos x ambientes. In: RAMALHO, M. A. P.; SANTOS, J. B. dos.; ZIMERMANN, M. J. O. Genética quantitativa em plantas autógamas: aplicação no melhoramento do feijoeiro. Goiânia : Editora da UFG, 1993. p. 131-169. (Publicação, 120).

SCAPIM, C. A.; CARVALHO, C. G. P. de; CRUZ, C. D. Uma proposta de classificação dos coeficientes de variação para a cultura do milho. Pesquisa Agropecuária Brasileira, Brasília, v. 30, n. 5, p. 683-686, maio 1995. 